heterosexual populations are not well understood. Objective was to describe female clients and partners of MSM sex workers (MSM$\mathrm{SW}$ ) in Lagos and to determine risk factors for unprotected sex between women and MSM-SW.

Methods MSM were recruited through respondent driven sampling, a novel methodology designed to access hidden populations. MSMSW were identified through self-reporting and consent was obtained. Participants completed a pre-tested questionnaire and a behaviour diary. The questionnaire included questions on details of each sexual partner for 4 weeks; each sexual encounter with each partner; and the three most recent partnerships. Data were summarised as proportions and compared using chi square test.

Results $62 \mathrm{MSM}-\mathrm{SW}$ participated in the study and concluded the selfreported questionnaire and behaviour diary. MSM-SW recorded 311 partners, out of which $66(21.2 \%)$ were female and aged between 16 and 38 years. Thirty-two $(10.3 \%)$ were recent female partners. Of the recent female partners, 17 (53.1\%) were unmarried, $14(43.8 \%)$ married and one was a spouse of MSM-SW. 60 (90.1\%) of female partners of MSM-SW paid or were paid for sex (39/60,65\% paid MSMSW while 21/60, 35\% were paid by them). There were 52 single episode sexual contacts and 14 regular partnerships. Of the 50 new transactional contacts, more than half $(56 \%)$ met in the bars, restaurants, clubs, motels and beaches. All the sexual encounters between MSM-SW and their female partners involved penetrative sex-vaginal sex (VS)(53, 80\%); anal sex (AS) (39, 59\%); and both (31, $47 \%)$. Unprotected sex was significantly more frequently associated with sex with regular partner than with single episode sexual contact $(79 \%$ vs $52 \% ; \mathrm{p}=0.02, \mathrm{OR}=3.0)$ and non-transactional sex than sex paid for by the female partners ( $71 \%$ vs $46 \%$; $p=0.029, \mathrm{OR}=2.8$ ).

Conclusion MSM-SW in Nigeria have a lot of female clients and partners and frequently engage in transactional and unprotected sex with them. Majority of these female partners are in heterosexual married relationships. Understanding these interactions between MSM and heterosexual populations is very crucial to any HIV intervention targeted on MSM and by extension the general population.

\section{P1-S2.11 HIGH PREVALENCE OF HIV, OTHER SEXUALLY TRANSMITTED INFECTIONS AND RISK PROFILE IN MALE COMMERCIAL SEX WORKERS WHO HAVE SEX WITH MEN IN THE NETHERLANDS}

doi:10.1136/sextrans-2011-050108.68

W J Cuypers, A M Niekamp, R Keesmekers, L Spauwen, D Hollman, D Telg, N DukersMuijrers, C Hoebe. Public Health Service, Southern Limburg, Geleen, Netherlands

Background Little is known about the epidemiology and burden of sexually transmitted infections (STI) in male commercial sex workers who have sex with men (MSM-CSWs) in the Netherlands and other Western countries. MSM-CSWs are often hard to reach for prevention and STI testing. This feasibility study aimed to reach MSM-CSWs, assess their socio-demographics and prevalence of HIV and other STI, and their risk profile.

Methods Retrospective, cross sectional study. Demographic and STI diagnosis data were retrieved from standardised medical records of MSM-CSWs consulted during outreach activities by a public health service in Southern Limburg, The Netherlands (2009 and 2010). Chlamydia trachomatis (CT) and Neisseria gonorrhoeae (NG) were diagnosed by NAAT and serology was performed for HIV (HIV-Ag/ $\mathrm{Ab}$ ), syphilis (TPPA, FTA-ABS, VDRL) and hepatitis B (anti-HBc and HBsAg).

Results In 2009 and 2010139 consultations by 99 MSM-CSW were performed. Their median age was 23.6 years; $56 \%$ of participants were of Romanian nationality, $20 \%$ of other (East) European nationality, $16 \%$ of South American nationality and $8 \%$ of Dutch nationality. Of participants, 50\% self identified as homosexual, 33\% as bisexual and $17 \%$ as heterosexual. All MSM-CSWs were tested for STI. Resultsof testing at first time consultation revealed that $45.5 \%$ was diagnosed with at least one STI-infection with CT was most prevalent (18.2\%), followed by infection with NG (12.1\%) and HIV (11.1\%). Of participants, $24.2 \%$ had evidence of exposure to Treponema pallidum; half was infectious at time of diagnosis. Of participants, $28.3 \%$ had evidence of previous exposure to hepatitis B virus (anti-HBc positive); $8.1 \%$ was infectious at time of diagnosis (HBsAg positive). 24.2\% was fully vaccinated against hepatitis B.

Conclusions Identification and recruitment for STI testing and prevention of this population at high risk for HIV and STI is shown to be feasible. Follow-up and retention appears to be a challenge. MSM-CSWs who have sex with men pose a serious STI acquisition and transmission risk regarding MSM-CSWs users. Further research is urgently required to address the needs of MSM-CSWs and explore prevention strategies. Furthermore, targeted health education to encourage safer sex is needed.

\section{P1-S2.12 IMPROVING ACCESS TO STI CARE AMONG FEMALE SEX WORKERS BY STRENGTHENING SERVICE PROVIDER NETWORKS IN BANGALORE, SOUTH INDIA}

doi:10.1136/sextrans-2011-050108.69

B Shetti, P Sunil, P Bhattacharjee, S Moses, K H Prakash, M Vidyacharan. Karnataka Health Promotion Trust, Bangalore, India

Background The Karnataka Health Promotion Trust, in partnership with non-governmental and community-based organisations, has been implementing a focused HIV prevention project among female sex workers (FSWs) in Bangalore since 2005. Over 25000 distinct female sex workers have been contacted to date, and approximately 10000 distinct FSWs are regularly contacted each month. The majority of FSWs are street-based and home-based, and are dispersed throughout the city. In the first 3 years of the project, STI services were provided through eight program-linked clinics located in program drop-in-centres, and 20 external referral doctors. Discussions with FSWs indicated that distance and inconvenient timings of the clinics were major barriers to accessing STI services. A strategy was therefore developed in 2008 to expand the network of referral doctors. Referral doctor networks were established at sites based on recommendations made by FSWs, both in government facilities and in private clinics. A total of 70 referral doctors (preferred providers) were identified and trained to provide quality STI services. We evaluated the outcome of this changed strategy.

Methods Indicators related to STI services provided and their accessibility were extracted from the program's computerised management information system (CMIS).

Results Following the change in strategy, average monthly clinic visits by FSWs increased from $16 \%$ to $28 \%$ of the total available population. Quarterly clinic visits increased from 41\% in 2008 to $63 \%$ in 2010. Administration of presumptive STI treatment for newly identified FSWs increased from 35\% in 2008 to 58\% in 2010. Focus group discussions with FSWs indicated that they are more comfortable in accessing clinic services, as the preferred providers are closer to where they live and work, and are available at more convenient times, especially in the evenings. This resulted in the higher levels of service coverage. The sex workers also indicated that, although services currently are provided at no charge to them, they would continue to access them from the preferred providers if the project ended, even if they had to pay for the services.

Conclusions Establishing a network of referral doctors who have been identified and sanctioned by the FSW community has complemented program-linked STI clinic services in the context of a large-scale HIV prevention program in the city of Bangalore, and has made STI services more accessible. 